\title{
Koffein verbessert die Motorik beim Morbus Parkinson
}

Es gibt Hinweise darauf, dass Menschen, die sehr viel Kaffee trinken, signifikant seltener einen Morbus Parkinson entwickeln. Des Weiteren wissen wir, dass Koffein gegen Tagesmüdigkeit hilfreich ist. Tagesmüdigkeit wiederum ist bei Parkinsonpatienten häufig ein großes Problem, wobei dies sowohl durch das Krankheitsgeschehen im Mittelhirn als auch durch die Medikamente verursacht sein kann.

— In jüngster Zeit finden Adenosin-Rezeptor-Antagonisten als Therapeutika zunehmend Interesse im Rahmen der Parkinsonerkrankung. Koffein ist ein nicht selektiver Antagonist des Adenosinrezeptors, der wiederum interessante Verknüpfungen $\mathrm{zu}$ den motorischen Schleifen im Gehirn aufweist, die bei der Parkinson-Erkrankung defekt sind. In vielen Tierstudien konnte ein positiver motorischer Effekt von Koffein nachgewiesen werden. Dies gelang mittlerweile auch beim Menschen für Adenosin-2AAntagonisten wie zum Beispiel Preladenant.

Um zu prüfen, ob Koffein auch bei Patienten mit Parkinson-Erkrankung zu einer Verbesserung der Tagesmüdigkeit führt und ob Koffein zu einer Verbesserung der Motorik beiträgt, wurde eine sechswöchige randomisierte, placebokontrollierte Doppelblindstudie kreiert.

In dieser Studie wurde über sechs Wochen randomisiert entweder 100-200 mg Koffein zweimal täglich oder Placebo bei Parkinsonpatienten appliziert, die neben der gesicherten Diagnose eines idiopathischen Parkinson-Syndroms auch eine erhebliche Tagesmüdigkeit entsprechend des Epworth-SleepinessScale-Score $(\geq 10)$ aufweisen mussten.

Als primärer Endpunkt wurde die Verbesserung der Epworth-SleepinessScale gewählt, wobei Koffein eine nicht signifikante Verbesserung diesbezüglich erzielte. Im Clinical Global Impression of Change Score fand sich dagegen eine Verbesserung der Somnolenz. Interessanterweise kam es unter Koffein zu einer signifikanten Verbesserung der Unified Parkinson Disease Rating Scale (UPDRS) bis um sechs Punkte im Schnitt nach der sechsten Woche, wobei in der UPDRS Teil III davon drei Punkte

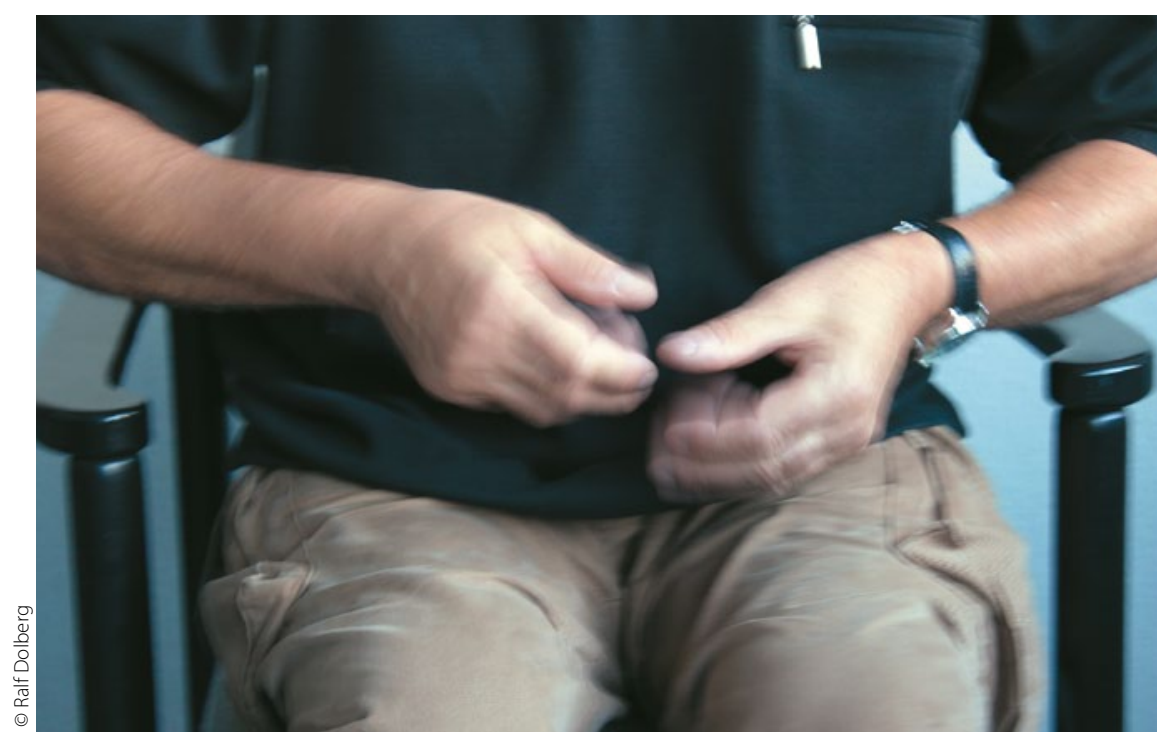

Parkinson: Bessern zwei bis drei Tassen Kaffee am Tag die Symptome? erreicht wurden. Lebensqualität, Depression oder Schlafqualität veränderten sich in dieser Studie weder im Placebonoch im Verum-Arm. Bezüglich der $\mathrm{Ne}$ benwirkungen gab es keine signifikanten Unterschiede zwischen den Gruppen.

In dieser Pilotstudie konnten ein nennenswerter Koffeineffekt bezüglich der Motorik und eine leichte Verbesserung der Tagesmüdigkeit nachgewiesen werden. Diese überraschend positiven Ergebnisse belegen somit, dass Adenosin-A2-Rezeptor-Antagonisten ein möglicherweise hohes therapeutisches Potenzial haben und dass eine längere und mit mehr Patienten bestückte zweite Studie zur Koffeintherapie bei Parkinsonpatienten gerechtfertigt ist.

\section{- R. B. Postuma et al.}

Caffeine for treatment of Parkinson disease: A randomized controlled trial. Neurology 2012; 79: $651-658$

\section{Kommentar}

Diese Studie eröffnet ein interessantes neues Feld und ist nach den eher enttäuschenden Ergebnissen des ersten Adenosin-A2Antagonisten, Istradefyllin, zusammen mit Daten des neuesten Produkts Preladenant der Beginn für interessante klinische Studien zur Wirksamkeit von Adenosin-RezeptorAntagonisten bei Patienten mit ParkinsonErkrankung.

Insbesondere dem Koffein sollten wir somit mehr Aufmerksamkeit schenken. Zu prüfen ist, ob diese 61 Patienten uns schon ermutigen sollten, Parkinsonpatienten, die noch keinen Kaffee zu sich nehmen, dies eindringlich zu empfehlen. Für den Leser mag es interessant sein zu erfahren, dass in einer 150 ml Tasse Bohnenkaffee, die im Filter-AufgussVerfahren gebrüht wird, durchschnittlich 80 mg Koffein enthalten sind.

H. REICHMANN 